\title{
Welche Medikamente sind in der Stillzeit erlaubt?
}

\author{
Eine Übersicht für den Anästhesisten, Geburtshelfer und Kinderarzt
}

\author{
Arabella Fischer (D) - Clemens Ortner - Thomas Hartmann · Stefan Jochberger · Klaus Ulrich Klein
}

Eingegangen: 2. November 2017 / Angenommen: 11. April 2018 / Online publiziert: 24. April 2018

(c) Der/die Autor(en) 2018

\begin{abstract}
Zusammenfassung Oft liegen abweichende oder keine pharmakokinetischen Daten für Medikamente zur Anwendung in der Stillzeit vor. Fachinformationen empfehlen eine Stillpause für $24 \mathrm{~h}$ nach Medikamenteneinnahme, um sich rechtlich abzusichern, was jedoch in der klinischen Praxis nicht mehr zeitgemäß ist. Die Mutter soll unmittelbar nach der Geburt zum frühen Stillen animiert werden. Gleichzeitig soll hierbei die Medikamentenexposition über die Muttermilch für das Neugeborene gering gehalten werden. Pharmakokinetische Daten und klinische Erfahrungswerte der wichtigsten Medikamente für die Anwendung in der Stillzeit sind in diesem Artikel zusammengefasst. Als Faustregel kann eine Mutter nach einer Anästhesie wieder stillen, sobald sie genügend wach ist, um ihr Kind anzulegen.
\end{abstract}

Dr. med. univ. A. Fischer, MD ( $\square)$.

Ass. Prof. PD Dr. C. Ortner, MD .

Ass. Prof. Dr. med. univ. T. Hartmann, MD .

Assoc. Prof. PD Dr. K. U. Klein, MD

Universitätsklinik für Anästhesie, Allgemeine

Intensivmedizin und Schmerztherapie, Medizinische

Universität Wien, Währinger Gürtel 18-20, 1090 Wien,

Österreich

arabella.fischer@meduniwien.ac.at

Ass. Prof. PD Dr. C. Ortner, MD

clemens.ortner@meduniwien.ac.at

Ass. Prof. Dr. med. univ. T. Hartmann, MD

thomas.hartmann@meduniwien.ac.at

Assoc. Prof. PD Dr. K. U. Klein, MD

ulrich.klein@meduniwien.ac.at

PD Dr. S. Jochberger

Universitätsklinik für Anästhesie und

Intensivmedizin, Medizinische Universität Innsbruck,

Anichstraße 35, 6020 Innsbruck, Österreich

stefan.jochberger@i-med.ac.at
Schlüsselwörter Geburtshilfliche Anästhesie · Postpartal · Stillzeit · Medikamente

Which medications are safe while breastfeeding? A synopsis for the anesthetist, obstetrician and pediatrician

Summary Pharmacokinetic data on drug administration during lactation are often inconsistent or missing. For legal reasons medicinal drug product information generally advises to interrupt breastfeeding for $24 \mathrm{~h}$ after medication intake. However this is not standard of care in clinical practice as the mother should be instructed to initiate breastfeeding as soon as possible after giving birth. At the same time the medication exposure over the breast milk for the newborn should be minimized. Aim of this article is to summarize pharmacokinetic data and to give important clinical information on medications frequently administered during the lactation period. As a general rule a mother can start breastfeeding following anesthesia as soon as she is able to get her baby latched on her breast.

Keywords Obstetric anesthesia - Postpartum · Lactation period $\cdot$ Medication

\section{Einleitung}

Stillen bietet viele Vorteile für Kind und Mutter. Das Kind erhält eine Immunität und eine optimale Ernährung. Das Risiko für den plötzlichen Kindstod ist bei gestillten Kindern reduziert [1]. Bei der Mutter führt es zu einer Verkleinerung der Gebärmutter, einem reduzierten Risiko für Brust- und Eierstockkrebs, Diabetes und Hypertonie $[2,3]$.

Während des Stillens kommt es zur Ausschüttung von Oxytocin, was auch schmerzhafte Bauchkrämpfe nach vaginaler oder Kaiserschnitt-Geburt verstärkt [4]. 
Eine effektive Schmerztherapie nach vaginaler und Kaiserschnittentbindung ist von zentraler Bedeutung, um eine frühe Mobilisation postpartal zu gewährleisten. Unter anderem setzt bei verstärktem postpartalen Schmerz die Milchproduktion verzögert ein [5]. Mütter mit geringen Schmerzen nach Kaiserschnitt stillen ihre Neugeborenen früher und häufiger [5, 6]. Oral, intravenös, spinal oder epidural verabreichte Medikamente zur Anästhesie und Analgesie diffundieren in die Muttermilch. Leider fehlen pharmakokinetische Daten für einige Medikamente zur Anwendung in der Stillzeit oder es liegen abweichende Daten vor [7, 8]. Um sich rechtlich abzusichern, empfehlen die Fachinformationen fast aller Medikamente eine Stillpause für $24 \mathrm{~h}$ nach Medikamenteneinnahme und gar Verwerfen der in dieser Zeit gebildeten Milch [9]. Dies gilt jedoch in der klinischen Praxis als veraltet [9]. Anästhesisten, Geburtshelfer und Kinderärzte stehen vor der Herausforderung, die Mutter zum frühen Stillen zu animieren und dabei gleichzeitig die Medikamentenexposition über die Muttermilch für das Neugeborene zu minimieren. Viele Mütter sind verunsichert bei Medikamenteneinnahme während der Stillzeit, was zur Stillpausen oder Verweigerung der Medikamenteneinnahme führt [10].

Der Artikel fasst die pharmakokinetischen Daten aus Studien und klinischen Erfahrungswerte für die wichtigsten Medikamenten in der Stillzeit, insbesondere in den ersten Tagen nach der Geburt zusammen.

\section{Zusammensetzung der Muttermilch}

Die Milch in den ersten 48 bis 72 Stunden nach der Geburt heißt Kolostrum und ist proteinreich sowie fettarm. Bis 72 Stunden nach der Geburt werden nur geringe Mengen an Kolostrum vom Neugeborenen aufgenommen. Nach etwa 5 Tagen wird die eigentliche Muttermilch produziert. Bei voller Brust fließt eine wässrige Vordermilch, bei sich leerender Brust fließt eine fettreiche Nachmilch. Der Fettgehalt der Muttermilch variiert nicht nur von Vorder- zur Nachmilch, sondern auch im Verlauf des Tages und von Brust zu Brust [2].

\section{Diffusion von Medikamenten in die Muttermilch}

Der Milch-Plasma-Quotient (M/P-Quotient) ist das Verhältnis der Konzentration einer Substanz in der Muttermilch zum mütterlichen Plasma. Die orale Bioverfügbarkeit und Halbwertszeit (HWZ) der Substanz bestimmen den Plasmaspiegel beim Säugling. Die Halbwertszeit vieler Medikamente ist bei Neonaten länger als bei Erwachsenen [9].

Die relative Dosis oder relative kindliche Dosis ist das Verhältnis der über die Muttermilch aufgenommene Menge einer Substanz (in mg pro kg Körpergewicht des Säuglings) und der Menge der Substanz, die die Mutter erhalten hat (in mg pro kg Körpergewicht der Mutter) [9, 11]. Prinzipiell gilt ein Medika- ment mit einer relativen Dosis unter $10 \%$ als sicher für die Anwendung während der Stillzeit. Bei einer relativen Dosis zwischen 10 und 25\% ist Vorsicht bei der Anwendung während der Stillzeit geboten. Bei einer relativen Dosis über $25 \%$ ist die Anwendung während der Stillzeit kontraindiziert [12].

Nicht-ionisierte Substanzen können über passive Diffusion durch Zellmembranen in die Muttermilch gelangen [9, 13]. Auch Basen oder Säuren, die sowohl im nicht- und ionisierten Zustand vorliegen, können im nicht-ionisierten Zustand durch Zellmembranen gelangen. Die Muttermilch hat einen niedrigeren $\mathrm{pH}$ Wert als das Plasma [9]. Substanzen mit einem hohen pKa-Wert (d.h. starke Basen) diffundieren im nicht-ionisierten Zustand durch Zellmembranen in die Muttermilch, werden im sauren Milieu der Muttermilch mit einem $\mathrm{H}+$ geladen und können im ionisierten $\mathrm{Zu}$ stand schwieriger durch die Muttermilch ins mütterliche Plasma rückdiffundieren (Ionenfalle) [9, 13]. Der pKa-Wert (aus dem Englischen acid=Säure) ist der negative dekadische Logarithmus der Säurekonstante Ka. Je kleiner der pKa-Wert ist, desto stärker ist die Säure. Zusammenfassend diffundieren Substanzen mit niedriger molarer Masse, geringer Proteinbindung, niedrigem Ionisationsgrad, langer Halbwertszeit, hoher oraler Bioverfügbarkeit, hoher Lipidlöslichkeit und Substanzen mit hohem pKa-Wert leicht in die Muttermilch [2, 9, 13].

\section{Risikofaktoren für verzögerte oder gestörte Milch-} produktion

Erstgebärende haben ein erhöhtes Risiko für eine verzögerte Laktogenese [14].

Neugeborene unter einem Gewicht von $3,6 \mathrm{~kg}$ haben möglicherweise eine noch nicht voll entwickelte Saugfähigkeit, was auch zu einer verzögerten Milchproduktion führen kann $[15,16]$.

Überdurchschnittliche Geburtsanstrengungen und ein unvorhergesehener Kaiserschnitt sind weitere Risikofaktoren [14, 15]. Mütter, die eine Spinal-, Epiduralanästhesie oder sonstige Analgetika vor vaginaler Geburt oder Kaiserschnitt erhalten, haben ein höheres Risiko für ein verspätetes Einsetzen der Milchproduktion als jene, die keinerlei Anästhesie oder Analgesie vor vaginaler Geburt erhalten [17]. Die aufgenommene Dosis an Anästhetika oder Analgetika über die Plazenta könnte die Saugfähigkeit des Neugeborenen einschränken [17]. Eine Epiduralanästhesie reduziert auch die Oxytocinproduktion [18]. Adipöse Gebärende erleben oft längere Geburten und haben ausgeprägtere postpartale Ödeme, die das Einsetzen der Laktation verzögern können [14-16, 19, 20]. Insulin regelt den Transport der Nährstoffe zur Brustdrüse [14]. Ein mütterlicher Diabetes kann somit ein verspätetes Einsetzen der Milchproduktion hervorrufen [14, 16, 21, 22]. Hypertonie in der Schwangerschaft kann die Laktogenese verzögern und beeinflusst auch die chemische Zusammensetzung der Muttermilch [23]. 
Tab. 1 Anästhetika in der Stillzeit

\begin{tabular}{|c|c|c|c|c|c|c|}
\hline \multicolumn{7}{|l|}{ Anästhetika } \\
\hline & $\begin{array}{l}\text { Relative } \\
\text { Dosis [\%] }\end{array}$ & $\begin{array}{l}\text { Milch (Kolos- } \\
\text { trum)-Plasma- } \\
\text { Quotient }\end{array}$ & $\begin{array}{l}\text { Orale } \\
\text { Biover- } \\
\text { fügbarkeit }\end{array}$ & Pharmakokinetische Daten & $\begin{array}{l}\text { Negative klinische } \\
\text { Effekte auf Kind } \\
\text { oder Mutter }\end{array}$ & $\begin{array}{l}\text { Klinische } \\
\text { Empfehlung }\end{array}$ \\
\hline Propofol $[2,7,13]$ & 0,025 & $<1$ & Gering & $\begin{array}{l}\text { Schnelle Ausscheidung; Proteinbindung } 99 \% \text {; } \\
\text { HWZ im mütterlichen Plasma: } 1 \mathrm{~h}\end{array}$ & Keine & Sicher \\
\hline Thiopental $[2,7]$ & $2-3$ & 0,6 & n.a. & $\begin{array}{l}\text { Proteinbindung } 60-96 \% \text {; HWZ im mütterlichen } \\
\text { Plasma: } 12 \mathrm{~h}\end{array}$ & Keine & Sicher \\
\hline Etomidat $[2,8,13,62]$ & - & $0-1,2^{\mathrm{a}}$ & n.a. & $\begin{array}{l}\text { Schnelle Ausscheidung; HWZ im mütterlichen } \\
\text { Plasma: } 5 \mathrm{~h}\end{array}$ & Keine & Sicher \\
\hline Ketamin $[2,8,62]$ & - & - & $20 \%$ & HWZ im mütterlichen Plasma: 2-3h & Keine & Sicher \\
\hline Dexmedetomidin $[8,9]$ & $0,04-0,098$ & - & n.a. & HWZ im mütterlichen Plasma: 5h & Keine & Sicher \\
\hline $\begin{array}{l}\text { Sevofluran, Desfluran, } \\
\text { Isofluran }[2,8,13]\end{array}$ & - & - & Gering & Schnelle Ausscheidung & Keine & Sicher \\
\hline Lachgas $\left(\mathrm{N}_{2} \mathrm{O}\right)[2,8]$ & - & - & n.a. & $\begin{array}{l}\text { Schnelle Ausscheidung; HWZ im mütterlichen } \\
\text { Plasma }<3 \text { min }\end{array}$ & Keine & Sicher \\
\hline Xenon $[2,8]$ & - & $0^{b}$ & n.a. & n.a. & Keine & Sicher \\
\hline
\end{tabular}

Schilddrüsenhormone beeinflussen die Verfügbarkeit von Lipiden für die Muttermilch und den Aufbau des epithelialen Brustgewebes [24]. Hypothyreose ist somit ein weiterer Risikofaktor [14, 16, 24]. Postpartale Blutungen sowie ein Hämoglobinwert unter $12 \mathrm{~g} / \mathrm{dl}$ sind mit einer verzögerten Milchproduktion assoziiert $[14,16]$. Allerdings haben fast alle Schwangere um den Geburtstermin und fast alle Wöchnerinnen einen Hämoglobinwert unter $12 \mathrm{~g} / \mathrm{dl}$. Die Zunahme des Plasmavolumens in der Schwangerschaft bedingt einen Abfall der Hämoglobinkonzentration auf durchschnittlich $11 \mathrm{~g} / \mathrm{dl}$ in der 36. Schwangerschaftswoche [25]. Beim Sheehan Syndrom kommt es durch einen postpartalen hämorrhagischen Schock zu einer ischämischen Nekrose der Hypophyse mit Ausbleiben der Laktation und in weiterer Folge Ausbleiben der Menses [26]. Von 35.588 neuseeländischen Frauen nach postpartaler Blutung, die keine Blutkonserven erhielten, stillten $88 \%$ bei Spitalsentlassung. Von 4561 Frauen nach postpartaler Blutung, die Blutkonserven erhielten, stillten $82 \%$ bei Spitalsentlassung [27]. Die Indikation einer Bluttransfusion sollte natürlich nicht zur Förderung der Laktogenese gestellt werden [27]. Prinzipiell ist bei stationären und hämodynamisch stabilen Patienten erst unter einem Hämoglobinkonzentration von $7 \mathrm{~g} / \mathrm{dl}$ die Gabe von Erythrozythenkonserven zu empfehlen laut AABB (American Association of Blood Banks) [28]. Es gibt derzeit keine Evidenz, ob das unmittelbare Stillen oder eine Brustwarzenstimulation und die damit verbundene Oxytocinausschüttung das Ausmaß der Blutung in der Zeit zwischen Kindesgeburt und Nachgeburt beeinflusst [29].

\section{Medikamente in der Stillzeit}

\section{Anästhetika (Tab. 1)}

Injektionsanästhetika sind lipophil und diffundieren schnell vom Plasma in die Muttermilch. Dank ihrer kurzen Halbwertszeit und raschen Umverteilung sinkt die Plasmakonzentration, was eine Rückdiffusion der Anästhetika von der Muttermilch ins Plasma bewirkt [9].

\section{Opioide (Tab. 2)}

Ein Opioidkonsum während der Schwangerschaft wirkt sich auf das Neugeborene aus [30]. Bei Transfer von Opiaten über die Plazenta kann sich nach der Geburt zum einen ein neonatales Abstinenzsyndrom zeigen, welches mit neurologischen Symptomen und gastrointestinalen Dysfunktionen einhergeht [30, 31]. Zum anderen kann es beim Neugeborenen nach fetaler Opioidexposition zu einer Toleranz gegenüber Opiaten kommen, da es zu einer Downregulation der Opioidrezeptoren kommt [30]. Neugeborene, die bereits im Uterus opioidexponiert waren, haben somit eine erhöhte Toleranz [32].

Neugeborene haben eine deutlich längere Halbwertszeit für viele Opioide (Fentanyl, Alfentanil, Pethidin) als Erwachsene [7]. Insbesondere wenn keine Daten zum Medikamententransfer in die Muttermilch vorliegen, sollte bei jeder stillenden Mutter, die Opioide bekommt, das Kind auf Zeichen von Atemdepression, Sedierung und herabgesetzter Aufmerksamkeit beobachtet werden.

Bei stillenden Müttern können intravenöse Einzeldosen von Fentanyl, transdermale Fentanylpflaster oder epidural appliziertes Fentanyl sicher angewandt werden [2]. Der Milch/Plasma Quotient bei 13 stillen- 
Tab. 2 Opiate in der Stillzeit

\begin{tabular}{|c|c|c|c|c|c|c|}
\hline \multicolumn{7}{|l|}{ Opioide } \\
\hline & $\begin{array}{l}\text { Relative } \\
\text { Dosis } \\
{[\%]}\end{array}$ & $\begin{array}{l}\text { Milch (Kolos- } \\
\text { trum)-Plasma- } \\
\text { Quotient }\end{array}$ & $\begin{array}{l}\text { Orale } \\
\text { Biover- } \\
\text { fügbarkeit }\end{array}$ & Pharmakokinetische Daten & $\begin{array}{l}\text { Negative klinische } \\
\text { Effekte auf Kind oder } \\
\text { Mutter }\end{array}$ & $\begin{array}{l}\text { Klinische } \\
\text { Empfehlung }\end{array}$ \\
\hline Morphin $[2,13,33]$ & $0,8-12$ & $1,1-3,6$ & $26 \%$ & $\begin{array}{l}\text { HWZ in Muttermilch: } 3 \mathrm{~h} \text {; renale Elimina- } \\
\text { tion von Morphin-6-Glucuronid (aktiver } \\
\text { Metabolit) }\end{array}$ & Keine bei Einzeldosis & Vorsicht \\
\hline $\begin{array}{l}\text { Hydromorphon }[2,8, \\
13,36]\end{array}$ & 0,67 & 2,57 & $32 \%$ & HWZ in Muttermilch: $10,5 \mathrm{~h}$ & Keine bei Einzeldosis & Vorsicht \\
\hline Hydrocodon $[2,8,36]$ & $0,2-9$ & - & $60-70 \%$ & $\begin{array}{l}\text { Durch hepatisches mikrosomales Enzym } \\
\text { CYP2D6 zu Hydromorphon metabolisiert }\end{array}$ & ZNS Depression & Vorsicht \\
\hline $\begin{array}{l}\text { Remifentanil [2, 7, } 8 \text {, } \\
\text { 13] }\end{array}$ & - & - & Gering & $\begin{array}{l}\text { Proteinbindung } 70 \% \text {; Metabolisierung } \\
\text { durch Plasmacholinesterasen; HWZ im } \\
\text { mütterlichen Plasma: 3-10 min }\end{array}$ & Keine & Sicher \\
\hline $\begin{array}{l}\text { Codein } \\
{[2,7-9,13,33,36]}\end{array}$ & 7,9 & $1,3-2,5$ & $100 \%$ & $\begin{array}{l}\text { HWZ im mütterlichen Plasma: } 3 \mathrm{~h} \text {; sehr } \\
\text { lipophil; durch hepatisches mikrosomales } \\
\text { Enzym CYP2D6 zu Morphin metabolisiert }\end{array}$ & $\begin{array}{l}\text { Bei genetisch beding- } \\
\text { ten ultraschnellen } \\
\text { CYP2D6 Metabolismus } \\
\text { (in 1,4\% der west- } \\
\text { europäischen Mütter): } \\
\text { schwere neonatale } \\
\text { Atemdepression }\end{array}$ & Kontraindiziert \\
\hline Oxycodon $[2,7,9,36]$ & 3 & 3,42 & $50 \%$ & $\begin{array}{l}\text { Durch hepatisches mikrosomales Enzym } \\
\text { CYP2D6 metabolisiert; } \\
\text { HWZ im mütterlichen Plasma: } 4-6 \mathrm{~h} \text {; } \\
\text { Proteinbindung } 40 \%\end{array}$ & ZNS Depression & Kontraindiziert \\
\hline Tramadol $[2,7]$ & 0,64 & 0,1 & $60-75 \%$ & $\begin{array}{l}\text { Durch hepatisches mikrosomales Enzym } \\
\text { CYP2D6 metabolisiert; } \\
\text { HWZ im mütterlichen Plasma: } 6 \text { h; Protein- } \\
\text { bindung } 20 \%\end{array}$ & Keine & Sicher \\
\hline $\begin{array}{l}\text { Pethidin }[2,7,9,13,33 \text {, } \\
\text { 41] }\end{array}$ & 4 & $0,82-1,59$ & $<50 \%$ & $\begin{array}{l}\text { HWZ im mütterlichen Plasma: } 13 \mathrm{~h} \text {; HWZ } \\
\text { im mütterlichen Plasma des aktiven } \\
\text { Metaboliten Norpethidin: } 65 \mathrm{~h}\end{array}$ & $\begin{array}{l}\text { ZNS Depression bei } \\
\text { intravenöser mütter- } \\
\text { licher Gabe }\end{array}$ & $\begin{array}{l}\text { Kontraindiziert; } \\
\text { Einzeldosis er- } \\
\text { fordert keine } \\
\text { Stillpause }\end{array}$ \\
\hline Fentanyl $[2,9,13,33]$ & 0,033 & 2,45 & Gering & $\begin{array}{l}\text { HWZ im mütterlichen Plasma: } 3 \mathrm{~h} \text {; rasche } \\
\text { renale Elimination }\end{array}$ & Keine bei Einzeldosis & Sicher \\
\hline Alfentanil $[2,7,9]$ & 4,6 & - & - & HWZ im mütterlichen Plasma: 1,6h & Keine & Sicher \\
\hline Sufentanil $[7,9]$ & - & - & Gering & $\begin{array}{l}\text { HWZ im mütterlichen Plasma: } 2,7 \mathrm{~h} \text {; } \\
\text { Proteinbindung } 92 \%\end{array}$ & Keine & Sicher \\
\hline Piritramid [7] & - & - & & HWZ im mütterlichen Plasma: 4-10h & Keine & Sicher \\
\hline Nalbuphin $[2,8,62]$ & $0,2-1,1$ & - & Gering & HWZ im mütterlichen Plasma: 3h & Keine & Sicher \\
\hline
\end{tabular}

den Frauen betrug für Fentanyl 2,45 [33]. Allerdings liegen keine Daten für mehrmalige intravenöse Dosen von Fentanyl vor [2]. Die Menge an Morphin in der Muttermilch kann ein Vielfaches über der oral, epidural oder intravenös verabreichten Morphinmenge im Plasmaspiegel der Mutter liegen. Die relative Dosis für Morphin bei epiduraler oder intravenöser Gabe bei 5 stillenden Müttern betrug <6\% [34]. Ein Fallbericht zeigt allerdings, dass die relative Dosis für Morphin bei oraler Gabe sogar bis zu 12\% hoch sein kann [35]. Daher ist erhöhte Vorsicht bei der Morphingabe bei stillenden Müttern geboten, auch wenn Einzeldosen keine negativen Auswirkungen auf das Kind zu haben scheinen [2]. Aufgrund der langen Halbwertszeit von Hydromorphon im mütterlichen Plasma und in der Muttermilch gilt vor allem bei wiederholten Gaben erhöhte Vorsicht bei stillenden Müttern [2]. Neugeborene sind besonders empfindlich für opiatinduzierte Effekte durch Hydrocodon [2]: Bei Neonaten ist die
Clearance von Hydrocodon herabgesetzt [36]. Ähnlich zu Codein, werden Hydrocodon, Oxycodon und Tramadol durch das hepatische mikrosomale Enzym CYP2D6 metabolisiert [37, 38]. Da es im Fall von Codein bei genetisch bedingtem ultraschnellen CYP2D6 Metabolismus zu schweren neonatalen Atemdepressionen kommen kann (Tab. 2), ist auch erhöhte Vorsicht bei Hydrocodon, Oxycodon und Tramadol geboten $[36,38]$. Es existieren zwei Fallberichte, in denen unter mütterlicher oraler Einnahme von Hydrocodon eine ZNS Depression (vermehrte Schläfrigkeit, Atemdepression) bei Säuglingen beschrieben ist [8, 39, 40]. Hierbei wurde nicht untersucht, ob die Mütter einen genetisch bedingten ultraschnellen CYP2D6 Metabolismus aufwiesen [39, 40]. Aufgrund der kurzen kontextsensitiven Halbwertszeit im mütterlichen Plasma von unter 10 min kann Remifentanil während der Geburt oder Sectio intravenös eingesetzt werden, eignet sich aber nicht zur postoperativen Analgesie 
Tab. 3 Benzodiazepine in der Stillzeit

\begin{tabular}{|c|c|c|c|c|c|c|}
\hline \multicolumn{7}{|l|}{ Benzodiazepine } \\
\hline & $\begin{array}{l}\text { Relative } \\
\text { Dosis } \\
{[\%]}\end{array}$ & $\begin{array}{l}\text { Milch (Kolos- } \\
\text { trum)-Plasma- } \\
\text { Quotient }\end{array}$ & $\begin{array}{l}\text { Orale } \\
\text { Biover- } \\
\text { fügbarkeit }\end{array}$ & Pharmakokinetische Daten & $\begin{array}{l}\text { Negative klinische } \\
\text { Effekte auf Kind oder } \\
\text { Mutter }\end{array}$ & $\begin{array}{l}\text { Klinische } \\
\text { Empfehlung }\end{array}$ \\
\hline $\begin{array}{l}\text { Diazepam } \\
{[2,7-9,50,51]}\end{array}$ & $3-13$ & $0,1-0,58$ & $99 \%$ & HWZ im mütterlichen Plasma: $43 \mathrm{~h}$ & $\begin{array}{l}\text { Benommenheit beim } \\
\text { Säugling }\end{array}$ & Vorsicht \\
\hline Lorazepam [2, 7, 8, 49] & $2,5-5$ & $0,15-0,26$ & $90 \%$ & HWZ im mütterlichen Plasma: $14 \mathrm{~h}$ & Keine & Sicher \\
\hline $\begin{array}{l}\text { Midazolam } \\
{[2,9,33]}\end{array}$ & 0,7 & $0,09-0,15$ & $27 \%$ & $\begin{array}{l}\text { HWZ im mütterlichen Plasma: } 2 \mathrm{~h} \text {; HWZ im } \\
\text { mütterlichen Plasma von aktiven Metaboli- } \\
\text { ten Hydroxymidazolam: } 5 \mathrm{~h}\end{array}$ & Keine & Sicher \\
\hline
\end{tabular}

[2]. Falls Pethidin der Schwangeren noch vor der Geburt verabreicht wird, kommt es beim Neugeborenen zu einer neonatalen Atemdepression [41]. Pethidin gilt nicht als Medikament der Wahl bei stillenden Müttern. Eine ZNS Depression im Sinne einer herabgesetzten Aufmerksamkeit wurde bei Säuglingen beobachtet, deren Mütter während der Stillzeit intravenös Pethidin erhielten $(n=5)$ im Vergleich $\mathrm{zu}$ jenen Müttern, die intravenös Morphin erhielten $(n=5)$ [42]. Bei epidural verabreichtem Pethidin nach elektiver Sectio zur postoperativen patientenkontrollierten Analgesie konnten bei 20 stillenden Müttern keine neonatale ZNS Depression beobachtet werden [43]. Weiters gibt es einen Fallbericht eines 10-jährigen Buben, der einen Krampfanfall nach postoperativer Pethidingabe erlitt [44]. Allerdings ist die Evidenzbasis, dass Norpethidin, der aktive Metabolit von Pethidin, die Krampfschwelle bei Erwachsenen oder Neugeborenen herabsetzt, karg [45].

\section{Benzodiazepine (Tab. 3)}

Wird schwangeren Frauen im Kreißsaal oder im geburtshilflichen Operationssaal vor der Geburt Diazepam verabreicht, haben ihre neugeborenen Kinder niedrige APGAR Scores [2]. Wird ihnen Lorazepam verabreicht, zeigen ihre Neugeborenen vermehrt Atemdepressionen laut einer randomisiert kontrollierten Studie (25 Erstgebärende, die Lorazepam erhielten, vs. 25 Erstgebärende, die ein Placebo erhielten) [46]. 42 Schwangere, die während der Sectio nach Abnabelung des Kindes zusätzlich zur epiduralen Anästhesie Lorazepam oder Diazepam intravenös erhielten, entwickelten ein Delir (Halluzinationen, Verwirrtheit, Unruhe, extremes Weinen, repetitive Handbewegungen) in $35 \%$ respektive $10 \%$ [47]. Zudem bewirkt vor allem Lorazepam eine anterograde Amnesie, die für 4 bis $8 \mathrm{~h}$ anhält $[47,48]$. In den USA können Ärzte geklagt werden, wenn sich die Mutter nicht mehr an die Geburt ihres Kindes als wichtiges Ereignis im Leben erinnert.

Bei stillenden Frauen gilt Midazolam als Prämedikation oder intraoperativ als sicher. Lorazepam, das eine mütterliche Plasmahalbwertszeit von 14 Stunden hat, kann stillenden Frauen sicher gegeben werden: Bei 124 Müttern, die Benzodiazepine während der
Stillzeit einnahmen (52\% der Mütter nahmen Lorazepam, 9\% nahmen Diazepam), konnten lediglich 2 Mütter vermehrte Schläfrigkeit bei ihren Säuglingen feststellen. Letztere nahmen eine höhere Anzahl an Benzodiazepine gleichzeitig ein [49]. 26\% aller Mütter zeigten unter Benzodiazepineinnahme Symptome von Verwirrtheit, vermehrter Müdigkeit, Übelkeit und Erbrechen [49]. Diazepam hat eine mütterlichen Plasmahalbwertszeit von 43 Stunden. Bei mütterlichen repetitiven Dosen während der Stillzeit akkumuliert Nordiazepam, der aktive Metabolit von Diazepam, im kindlichen Serum [8]. Bei 9 stillenden Müttern, die postpartal Diazepam einnahmen, konnte Diazepam im kindlichen Serum bis zu 10 Tage lang in beträchtlichen Dosen nachgewiesen werden [50]. Tatsächlich berichteten von insgesamt 246 stillenden Frauen jene, die Diazepam einnahmen $(n \leq 5)$, das Stillen pausiert zu haben, da sie vermehrte Schläfrigkeit bei ihren Säuglingen feststellten [51].

\section{Analgetika (Tab. 4)}

Nichtsteroidale Antirheumatika (NSAR) sind Medikamente der Wahl in der Stillzeit, da sie eine niedrige relative Dosis haben [7]. Geringe Dosen (100-300 mg/Tag regelmäßig) von Acetylsalicylsäure sind bei entsprechender mütterlicher Indikation (Präeklampsie, Thromboembolien, Herzklappenprothesen) in der Schwangerschaft und Stillzeit klar indiziert $[7,8,36,52]$. Die gelegentliche Einnahme bis zu maximal 1,5 g/Tag in der Stillzeit zur Analgesie ist berechtigt [7]. Allerdings ist eine regelmäßige hochdosierte Gabe von Acetylsalicylsäure in der Stillzeit kontraindiziert aufgrund der Möglichkeit einer metabolischen Azidose beim Säugling und der theoretischen Gefahr eines Kernikterus oder Reye-Syndrom beim Säugling [9, 52]. Die Einnahme von Paracetamol während der Schwangerschaft ist mit einem erhöhten Risiko für Verhaltensstörungen des Kindes 7 Jahre nach dessen Geburt assoziiert [53]. Diese Assoziation scheint nicht durch Verhaltensweisen oder soziale Faktoren innerhalb der Familie bedingt zu sein [53]. Allerdings wurde in der Studie von Stergiakouli et al. Dosis und Dauer der Einnahme von Paracetamol während der Schwangerschaft nicht berücksichtigt [53, 54]. Paracetamol ist weniger hepatotoxisch bei Neugeborenen als bei 


\section{übersicht}

Tab. 4 Analgetika in der Stillzeit

\begin{tabular}{|c|c|c|c|c|c|c|}
\hline \\
\hline Analgetika & $\begin{array}{l}\text { Relative } \\
\text { Dosis } \\
{[\%]}\end{array}$ & $\begin{array}{l}\text { Milch (Kolos- } \\
\text { trum)-Plasma- } \\
\text { Quotient }\end{array}$ & $\begin{array}{l}\text { Orale } \\
\text { Biover- } \\
\text { fügbarkeit }\end{array}$ & Pharmakokinetische Daten & $\begin{array}{l}\text { Negative klinische } \\
\text { Effekte auf Kind oder } \\
\text { Mutter }\end{array}$ & $\begin{array}{l}\text { Klinische } \\
\text { Empfehlung }\end{array}$ \\
\hline Diclofenac $[7,9,13]$ & 0,9 & - & $100 \%$ & $\begin{array}{l}\text { HWZ im mütterlichen Plasma: 1,8 } \mathrm{h} \text {; Prote- } \\
\text { inbindung } 99 \% \text {; geringe Lipidlöslichkeit }\end{array}$ & Keine & Sicher \\
\hline Ibuprofen $[7,9]$ & $<0,6$ & 0,008 & $80 \%$ & $\begin{array}{l}\text { HWZ im mütterlichen Plasma: 1,8-3,5h; } \\
\text { Proteinbindung 99\%; geringe Lipidlöslich- } \\
\text { keit }\end{array}$ & Keine & Sicher \\
\hline $\begin{array}{l}\text { Acetylsalicylsäure [7-9, } \\
33,52]\end{array}$ & $2-6$ & $0,05-0,1$ & $80-100 \%$ & $\begin{array}{l}\text { Lange HWZ; Proteinbindung } 80-90 \% \text {; } \\
\text { Kumulationsgefahr bei wiederholter Ein- } \\
\text { nahme }\end{array}$ & $\begin{array}{l}\text { Bei hoher regelmä- } \\
\text { Biger Dosis: metabo- } \\
\text { lische Azidose beim } \\
\text { Säugling }\end{array}$ & $\begin{array}{l}\text { Niedrige } \\
\text { Dosis sicher } \\
\text { (100-300 mg/Tag } \\
\text { regelmäßig oder } \\
\text { max. } 1,5 \mathrm{~g} / \mathrm{Tag} \\
\text { gelegentlich) }\end{array}$ \\
\hline $\begin{array}{l}\text { Celecoxib (selektiver } \\
\text { COX-2-Inhibitor) }[7,9]\end{array}$ & $<1$ & 0,23 & $99 \%$ & HWZ im mütterlichen Plasma: 8-12h & Keine & Sicher \\
\hline $\begin{array}{l}\text { Paracetamol }[7,9,33 \text {, } \\
\text { 36] }\end{array}$ & $6-12$ & $0,81-1$ & $>85 \%$ & $\begin{array}{l}\text { HWZ im mütterlichen Plasma: } 2,6 \mathrm{~h} \text {; Prote- } \\
\text { inbindung } 25 \%\end{array}$ & Keine & Sicher \\
\hline Metamizol [9] & - & 1 & $90 \%$ & $\begin{array}{l}\text { HWZ im mütterlichen Plasma: 1,8-4,6h; } \\
\text { Proteinbindung } 60 \%\end{array}$ & $\begin{array}{l}\text { Einzelner Fallbericht: } \\
\text { Zyanose des Säug- } \\
\text { lings }\end{array}$ & $\begin{array}{l}\text { Vorsicht; Einzeldo- } \\
\text { sis erfordert keine } \\
\text { Stillpause }\end{array}$ \\
\hline
\end{tabular}

Tab. 5 Muskelrelaxantien in der Stillzeit

\begin{tabular}{|c|c|c|c|c|c|c|}
\hline \multicolumn{7}{|c|}{ Muskelrelaxantien und Antagonisierung } \\
\hline & $\begin{array}{l}\text { Relative } \\
\text { Dosis } \\
{[\%]}\end{array}$ & $\begin{array}{l}\text { Milch (Kolos- } \\
\text { trum)-Plasma- } \\
\text { Quotient }\end{array}$ & $\begin{array}{l}\text { Orale } \\
\text { Biover- } \\
\text { fügbarkeit }\end{array}$ & Pharmakokinetische Daten & $\begin{array}{l}\text { Negative klinische } \\
\text { Effekte auf Kind oder } \\
\text { Mutter }\end{array}$ & $\begin{array}{l}\text { Klinische } \\
\text { Empfehlung }\end{array}$ \\
\hline $\begin{array}{l}\text { Succinylcholin }[2,8,9 \\
13]\end{array}$ & - & - & Gering & $\begin{array}{l}\text { HWZ im mütterlichen Plasma: } 3-5 \text { min; } \\
\text { große polarisierte Moleküle, geringe Lipid- } \\
\text { löslichkeit }\end{array}$ & Keine & Sicher \\
\hline $\begin{array}{l}\text { Nicht-depolarisierende } \\
\text { Muskelrelaxantien [2, 8, } \\
9,13]\end{array}$ & - & - & Gering & $\begin{array}{l}\text { Große polarisierte Moleküle, geringe Lipid- } \\
\text { löslichkeit }\end{array}$ & Keine & Sicher \\
\hline $\begin{array}{l}\text { Neostigmin } \\
{[2,7,13]}\end{array}$ & 0,1 & - & $1-2 \%$ & $\begin{array}{l}\text { HWZ im mütterlichen Plasma: 1,3h; } \\
\text { großes Molekül; Proteinbindung 15-25\% }\end{array}$ & $\begin{array}{l}\text { Vereinzelt abdomi- } \\
\text { nelle Krämpfe beim } \\
\text { Neugeborenen }\end{array}$ & Sicher \\
\hline $\begin{array}{l}\text { Sugammadex } \\
{[9,62]}\end{array}$ & - & - & & $\begin{array}{l}\text { HWZ im mütterlichen Plasma: } 2,5 \mathrm{~h} \text {; hohe } \\
\text { molare Masse, hydrophile Außenseite }\end{array}$ & Keine & Sicher \\
\hline
\end{tabular}

älteren Kindern, da erstere eine geringere Anzahl an Cytochrom-P450-Enzymen haben, die Paracetamol in toxische Metabolite umwandeln [36].

\section{Muskelrelaxantien (Tab. 5)}

Aufgrund der geringen Lipidlöslichkeit und geringen oralen Bioverfügbarkeit können nicht- und depolarisierende Muskelrelaxantien stillenden Mütter sicher verabreicht werden [2].

\section{Anticholinergika (Tab. 6)}

Aktuell gibt es keine publizierten Studien zur Anwendung von Atropin und Robinul bei stillenden Müttern $[2,7,8]$. Da im klinischen Alltag keine negativen Auswirkungen auf das Kind zu beobachten sind, gilt die
Verabreichung von Anticholinergika während der Stillzeit als sicher [2, 7, 8].

\section{Antiemetika (Tab. 7)}

Aufgrund fehlender sedativer Effekte können Ondansetron und Metoclopramid stillenden Müttern sicher verabreicht werden [2]. Die Einnahme von Droperidol während der Stillzeit führte in 2 Studien zu einer neurologischen und intellektuellen Beeinträchtigung der Neugeborenen [8]: Eine intellektuelle Beeinträchtigung wurde bei einem Säugling beobachtet, dessen Mutter während der Stillzeit Droperidol, aber auch Antipsychotika, ein Antiepileptikum, ein Benzodiazepin und ein Antidepressivum einnahm [55]. In einer randomisiert kontrollierten Studie erhielten Frauen nach Kaiserschnitt unter Regionalanästhesie zur postoperativen patientenkontrollierten Analgesie 
Tab. 6 Anticholinergika in der Stillzeit

\begin{tabular}{|c|c|c|c|c|c|c|}
\hline \multicolumn{7}{|l|}{ Anticholinergika } \\
\hline & $\begin{array}{l}\text { Relative } \\
\text { Dosis } \\
{[\%]}\end{array}$ & $\begin{array}{l}\text { Milch (Kolos- } \\
\text { trum)-Plasma- } \\
\text { Quotient }\end{array}$ & $\begin{array}{l}\text { Orale } \\
\text { Biover- } \\
\text { fügbarkeit }\end{array}$ & Pharmakokinetische Daten & $\begin{array}{l}\text { Negative klinische } \\
\text { Effekte auf Kind oder } \\
\text { Mutter }\end{array}$ & $\begin{array}{l}\text { Klinische } \\
\text { Empfehlung }\end{array}$ \\
\hline Atropin $[2,7]$ & - & - & $90 \%$ & $\begin{array}{l}\text { HWZ im mütterlichen Plasma: } 2-3 \mathrm{~h} \text {; Prote- } \\
\text { inbindung } 14-22 \%\end{array}$ & Keine & Sicher \\
\hline $\begin{array}{l}\text { Glycopyrroniumbromid } \\
{[2,8,13,63]}\end{array}$ & - & - & Gering & $\begin{array}{l}\text { HWZ im mütterlichen Plasma: } 2 \mathrm{~h} \text {; großes } \\
\text { Molekül }\end{array}$ & Keine & Sicher \\
\hline
\end{tabular}

Tab. 7 Antiemetika in der Stillzeit

\begin{tabular}{|c|c|c|c|c|c|c|}
\hline \multicolumn{7}{|l|}{ Antiemetika } \\
\hline & $\begin{array}{l}\text { Relative } \\
\text { Dosis } \\
{[\%]}\end{array}$ & $\begin{array}{l}\text { Milch (Kolos- } \\
\text { trum)-Plasma- } \\
\text { Quotient }\end{array}$ & $\begin{array}{l}\text { Orale } \\
\text { Biover- } \\
\text { fügbarkeit }\end{array}$ & Pharmakokinetische Daten & $\begin{array}{l}\text { Negative klinische } \\
\text { Effekte auf Kind oder } \\
\text { Mutter }\end{array}$ & $\begin{array}{l}\text { Klinische } \\
\text { Empfehlung }\end{array}$ \\
\hline $\begin{array}{l}\text { Metoclopramid }[2,7,9 \text {, } \\
\text { 33] }\end{array}$ & 4,7 & $0,5-4$ & $30-100 \%$ & $\begin{array}{l}\text { HWZ im mütterlichen Plasma: 2,6-4,6h; } \\
\text { Proteinbindung } 30 \%\end{array}$ & Keine & Sicher \\
\hline Ondansetron $[2,8]$ & - & - & $60 \%$ & $\begin{array}{l}\text { HWZ im mütterlichen Plasma: } 3,2-3,5 \mathrm{~h} \text {; } \\
\text { Proteinbindung } 70-76 \%\end{array}$ & Keine & Sicher \\
\hline Droperidol $[8,63]$ & - & - & & HWZ im mütterlichen Plasma: $2 \mathrm{~h}$ & $\begin{array}{l}\text { Neurologische Beein- } \\
\text { trächtigung }\end{array}$ & Vorsicht \\
\hline $\begin{array}{l}\text { H1-Rezeptor-Antagonis- } \\
\text { ten (Dimenhydrinat, } \\
\text { Diphenhydramin) }[8,9]\end{array}$ & - & - & $40-72 \%$ & $\begin{array}{l}\text { HWZ im mütterlichen Plasma 4-6h; Prote- } \\
\text { inbindung 78-99\% }\end{array}$ & $\begin{array}{l}\text { Keine; fallweise } \\
\text { Schläfrigkeit und } \\
\text { Koliken bei Neugebo- } \\
\text { renen }\end{array}$ & Sicher \\
\hline
\end{tabular}

Tab. 8 Lokalanästhetika in der Stillzeit

\begin{tabular}{|c|c|c|c|c|c|c|}
\hline \multicolumn{7}{|l|}{ Lokalanästhetika } \\
\hline & $\begin{array}{l}\text { Relative } \\
\text { Dosis } \\
{[\%]}\end{array}$ & $\begin{array}{l}\text { Milch (Kolos- } \\
\text { trum)-Plasma- } \\
\text { Quotient }\end{array}$ & $\begin{array}{l}\text { Orale } \\
\text { Biover- } \\
\text { fügbarkeit }\end{array}$ & Pharmakokinetische Daten & $\begin{array}{l}\text { Negative klinische } \\
\text { Effekte auf Kind oder } \\
\text { Mutter }\end{array}$ & $\begin{array}{l}\text { Klinische } \\
\text { Empfehlung }\end{array}$ \\
\hline Bupivacain $[2,7,9]$ & 1,15 & $0,34 \pm 0,24$ & $30 \%$ & $\begin{array}{l}\text { HWZ im mütterlichen Plasma: } 1,5-5,5 \mathrm{~h} \text {; } \\
\text { Proteinbindung 92-96\% }\end{array}$ & Keine & Sicher \\
\hline Lidocain $[2,7,9]$ & $1,8-4$ & $1,07 \pm 0,82$ & $35 \%$ & $\begin{array}{l}\text { HWZ im mütterlichen Plasma: } 1,5-2 \mathrm{~h} \text {; } \\
\text { Proteinbindung } 60-80 \%\end{array}$ & Keine & Sicher \\
\hline Ropivacain $[2,7,9]$ & - & $1,37 \pm 0,61$ & Gering & $\begin{array}{l}\text { HWZ im mütterlichen Plasma: } 1,8-4 \mathrm{~h} \text {; } \\
\text { Proteinbindung } 94 \%\end{array}$ & Keine & Sicher \\
\hline Mepivacain $[7,9]$ & - & - & & $\begin{array}{l}\text { HWZ im mütterlichen Plasma: } 1,9-3,2 \mathrm{~h} \text {; } \\
\text { Proteinbindung } 65-78 \%\end{array}$ & Keine & Sicher \\
\hline Articain $[7,9,62]$ & - & - & & $\begin{array}{l}\text { HWZ im mütterlichen Plasma: } 2 \mathrm{~h} \text {; Protein- } \\
\text { bindung } 95 \%\end{array}$ & Keine & Sicher \\
\hline Prilocain $[7,9]$ & - & - & & $\begin{array}{l}\text { HWZ im mütterlichen Plasma: } 1,5 \mathrm{~h} \text {; Prote- } \\
\text { inbindung } 55 \%\end{array}$ & $\begin{array}{l}\text { Bei höherer Dosie- } \\
\text { rung Methämoglobin- } \\
\text { bildung möglich }\end{array}$ & $\begin{array}{l}\text { Vorsicht; bei ge- } \\
\text { ringer Dosierung } \\
\text { keine Stillpause }\end{array}$ \\
\hline
\end{tabular}

Morphin intravenös $(n=20)$ oder Morphin mit Droperidol intravenös $(n=20)$ : Bei letzteren hatten die Säuglinge eine signifikante neurologische Beeinträchtigung [56].

\section{Lokalanästhetika (Tab. 8)}

Die lokale und neuroaxiale Verabreichung von Lokalanästhetika ist sicher, da Lokalanästhetika große polarisierende Moleküle sind, die nur schwer in die Milchdrüsengänge diffundieren können [2, 13]. Das ungeborene Kind nimmt während einer Epidural- anästhesie im Rahmen einer Sectio caesarea eine größere Menge an Lokalanästhetikum über die Plazenta auf als später über das Kolostrum [9]. Auch der Transfer von Lokalanästhestika über die Plazenta im Rahmen der Epiduralanästhesie für eine Sectio gilt als unbedenklich für das Kind: Während einer randomisiert kontrollierten Studie an 60 Gebärenden, die eine epidurale Anästhesie für eine Sectio erhielten (Ropivacain [ $n=30$ ] vs Bupivacain [ $n=30]$ ) war die Ratio von umbilikaler Venenkonzentration $\mathrm{zu}$ maternalen Venenkonzentration für Ropivacain und Bupivacain 0,72 respektive 0,69 [57]. Die Neugeborenen wiesen 
Tab. 9 Antibiotika in der Stillzeit

\begin{tabular}{|c|c|c|c|c|c|c|}
\hline \multicolumn{7}{|l|}{ Antibiotika } \\
\hline & $\begin{array}{l}\text { Relative } \\
\text { Dosis [\%] }\end{array}$ & $\begin{array}{l}\text { Milch (Kolos- } \\
\text { trum)-Plasma- } \\
\text { Quotient }\end{array}$ & $\begin{array}{l}\text { Orale } \\
\text { Biover- } \\
\text { fügbarkeit }\end{array}$ & Pharmakokinetische Daten & $\begin{array}{l}\text { Negative klinische } \\
\text { Effekte auf Kind oder } \\
\text { Mutter }\end{array}$ & $\begin{array}{l}\text { Klinische } \\
\text { Empfehlung }\end{array}$ \\
\hline Penizilline $[7,9]$ & $<1$ & $<1$ & $15-30 \%$ & $\begin{array}{l}\text { HWZ im mütterlichen Plasma: } 0,6-1 \mathrm{~h} \text {; } \\
\text { Proteinbindung }>60 \%\end{array}$ & $\begin{array}{l}\text { Fallweise dünner } \\
\text { Stuhlgang bei Säugling }\end{array}$ & Sicher \\
\hline Cefazolin $[8,63]$ & - & - & & HWZ im mütterlichen Plasma: $1,4 \mathrm{~h}$ & Keine & Sicher \\
\hline Clindamycin [7] & 1,6 & 0,47 & $>90 \%$ & $\begin{array}{l}\text { HWZ im mütterlichen Plasma: 2-3h; Prote- } \\
\text { inbindung } 60-90 \%\end{array}$ & Keine & Sicher \\
\hline Metronidazol $[7,9,33]$ & 12,6 & $0,9-2,13$ & $100 \%$ & $\begin{array}{l}\text { HWZ im mütterlichen Plasma: } 6-10 \mathrm{~h} \text {; } \\
\text { Proteinbindung }<20 \%\end{array}$ & $\begin{array}{l}\text { Vereinzelt weichere } \\
\text { Stuhlkonsistenz beim } \\
\text { Neugeborenen }\end{array}$ & Vorsicht \\
\hline Meropenem $[8,37,63]$ & $0,13-0,18$ & & & $\begin{array}{l}\text { HWZ im mütterlichen Plasma: } 1 \mathrm{~h} \text {; Protein- } \\
\text { bindung } 2 \%\end{array}$ & Keine & Sicher \\
\hline
\end{tabular}

Tab. 10 Sonstige Medikamente in der Stillzeit

\begin{tabular}{|c|c|c|c|c|c|c|}
\hline \multicolumn{7}{|c|}{ Sonstige Medikamente } \\
\hline & $\begin{array}{l}\text { Relative } \\
\text { Dosis } \\
{[\%]}\end{array}$ & $\begin{array}{l}\text { Milch (Kolos- } \\
\text { trum)-Plasma- } \\
\text { Quotient }\end{array}$ & $\begin{array}{l}\text { Orale } \\
\text { Biover- } \\
\text { fügbarkeit }\end{array}$ & Pharmakokinetische Daten & $\begin{array}{l}\text { Negative klinische } \\
\text { Effekte auf Kind oder } \\
\text { Mutter }\end{array}$ & $\begin{array}{l}\text { Klinische } \\
\text { Empfehlung }\end{array}$ \\
\hline Clonidin $[7,8]$ & $7-8$ & - & $65-100 \%$ & $\begin{array}{l}\text { HWZ im mütterlichen Plasma: } 10-20 \mathrm{~h} \text {; } \\
\text { Proteinbindung } 20-40 \%\end{array}$ & $\begin{array}{l}\text { Hoher Serumspiegel } \\
\text { beim Neugeboren bei } \\
\text { etwa } 2 / 3 \text { des mütter- } \\
\text { lichen Serumspiegel; } \\
\text { einzelner Fall mit } \\
\text { Schläfrigkeit beim } \\
\text { Neugeborenen }\end{array}$ & Vorsicht \\
\hline Prednisolon $[7,33]$ & $1-2$ & $0,05-0,25$ & $100 \%$ & $\begin{array}{l}\text { HWZ im mütterlichen Plasma: 2-3h; } \\
\text { Proteinbindung } 75-90 \%\end{array}$ & Keine & $\begin{array}{l}\text { Sicher (bei einma- } \\
\text { ligen mütterlichen } \\
\text { Dosen bis zu } 1 \mathrm{~g} \\
\text { pro Tag) }\end{array}$ \\
\hline Urapidil $[7,59]$ & - & - & $80-90 \%$ & $\begin{array}{l}\text { HWZ im mütterlichen Plasma: } 2-3 \mathrm{~h} \text {; } \\
\text { Proteinbindung } 80 \%\end{array}$ & - & $\begin{array}{l}\text { Vorsicht; kein Ab- } \\
\text { stillen erforderlich } \\
\text { bei präpartal be- } \\
\text { gonnener Therapie }\end{array}$ \\
\hline
\end{tabular}

5 min APGAR Scores $\geq 7$ sowie keine neurologischen Einschränkungen auf [57].

\section{Antibiotika (Tab. 9)}

Die Einnahme von Antibiotika während der Schwangerschaft verändert das maternale und fetale Darmmikrobiom [58]. Während der Stillzeit führt die Einnahme von Antibiotika zu einer veränderten Zusammensetzung der Muttermilch. Zum Beispiel ist der Gehalt an Bifidobakterien in der Muttermilch vermindert, was wiederum die Zusammensetzung des kindlichen Mikrobioms beeinflusst [58]. Das kindliche Mikrobiom ist essentiell für die Entwicklung des Immunsystems und beeinflusst, ob das Kind später adipös wird [58].

Penizilline und ältere Cephalosporine gehören in der Stillzeit zu den Antibiotika der Wahl [9].

\section{Sonstige Medikamente (Tab. 10)}

Clonidin sollte stillenden Müttern im Falle einer postpartalen Hypertonie nur mit Vorsicht verabreicht werden, da die relative Dosis hoch ist $[7,8]$. Bei postpartaler Neueinstellung der Hypertonie mit Urapidil sollte das Stillen unterbrochen werden, da keine pharmakokinetische Daten oder publizierten Erfahrungen zu Urapidil vorliegen [7, 8, 59]. Falls unter Antihypertensiva-Therapie ein Fortführen des Stillens erwünscht ist, können Labetalol, Dihydralazin, Nifedipin oder Nitroglycerin sicher verabreicht werden $[7,8,59,60]$. Alpha-Methyldopa ist das Antihypertensivum der ersten Wahl in der Schwangerschaft und kann in der Stillzeit sicher angewandt werden [7].

\section{Zusammenfassung}

Die relative Dosis der meisten Medikamente beim Säugling liegt selten über $10 \%$ (siehe Tab. 1-10).

Als Faustregel kann eine Mutter nach einer Anästhesie wieder stillen sobald sie genügend wach 
ist, um ihr Kind anzulegen. In der Regel birgt eine Stillunterbrechung ein größeres Risiko in sich als Medikamentenspuren in der Muttermilch. Jedoch ist insbesondere bei einigen Opioide, Diazepam, Droperidol, Clonidin und Urapidil Vorsicht geboten oder eine Kontraindikation während der Stillzeit gegeben (siehe Tab. 1-10). Analgetika der Wahl in der Stillzeit sind Paracetamol und NSAR. Für zusätzliche Sicherheit, insbesondere wenn Informationen wie relative Dosis oder klinische Erfahrung fehlen, sollten Säuglinge auf Zeichen von Atemdepression, Sedierung und herabgesetzter Aufmerksamkeit beobachtet werden $[2,13]$.

Um die Medikamentenaufnahme über die Muttermilch für den Säugling zu minimieren, empfiehlt es sich, dass die Mutter das Medikament im Augenblick des Stillens einnimmt. Bis zum nächsten Stillen ist dann für die meisten Medikamente die Halbwertszeit des Medikamentes vergangen [2].

$\mathrm{Zu}$ guter Letzt ist das ärztliche Personal angehalten, die klinische Empfehlung für die Gabe verschiedener Medikamente während der Stillzeit auf embryotox.de (http://www.embryotox.de) [7] oder LactMed ${ }^{\circledR}$ database (https://toxnet.nlm.nih.gov/newtoxnet/lactmed. htm) [8] oder http://www.motherisk.org [61] nachzuschlagen.

Funding Open access funding provided by Medical University of Vienna.

Interessenkonflikt A. Fischer, C. Ortner, T. Hartmann, S. Jochberger und K. U. Klein geben an, dass kein Interessenkonflikt besteht.

Open Access Dieser Artikel wird unter der Creative Commons Namensnennung 4.0 International Lizenz (http:// creativecommons.org/licenses/by/4.0/deed.de) veröffentlicht, welche die Nutzung, Vervielfältigung, Bearbeitung, Verbreitung und Wiedergabe in jeglichem Medium und Format erlaubt, sofern Sie den/die ursprünglichen Autor(en) und die Quelle ordnungsgemäß nennen, einen Link zur Creative Commons Lizenz beifügen und angeben, ob Änderungen vorgenommen wurden.

\section{Literatur}

1. Ip S, Chung M, Raman G, et al. Breastfeeding and maternal and infant health outcomes in developed countries. Evid Rep Technol Assess (Full Rep). 2007;153:1-186.

2. Dalal PG, Bosak J, Berlin C. Safety of the breast-feeding infant after maternal anesthesia. Paediatr Anaesth. 2014;359-71.https://doi.org/10.1111/pan.12331.

3. Chetwynd EM, Stuebe AM, Rosenberg L, et al. Cumulative lactation and onset of hypertension in African American women. Am J Epidemiol. 2017. https://doi.org/10.1093/ aje/kwx163.

4. Wen L, Hilton G, Carvalho B. The impact of breastfeeding on postpartum pain after vaginal and cesarean delivery. J Clin Anesth. 2015;33-8. https://doi.org/10.1016/j. jclinane.2014.06.010.

5. Dimitraki M, Tsikouras P, Manav B, et al. Evaluation of the effect of natural and emotional stress of labor on lactation and breast-feeding. Arch Gynecol Obstet. 2016;317-28. https://doi.org/10.1007/s00404-015-3783-1.
6. Hirose M, Hara Y, Hosokawa T, et al. The effect of postoperative analgesia with continuous epidural bupivacaine after cesarean section on the amount of breast feeding and infant weight gain. Anesth Analg. 1996;82:1166-9.

7. Charité Universitätsmedizin Berlin. Embryotox [Internet]. Einsehbar unter: http://www.embryotox.de. Zugegriffen: 24.01.2017.

8. U.S. National Library of Medicine. The LactMed ${ }^{\circledR}$ database [Internet]. Available from: https://toxnet.nlm.nih. gov/newtoxnet/lactmed.htm. Zugegriffen: 02.01.2018.

9. Nassen CA, Schaefer C, Wirbelauer J, et al. Anästhesie und Analgesie in der Stillperiode. Anaesthesist. 2014;415-21. https://doi.org/10.1007/s00101-014-2311-1.

10. Spiesser-RobeletL, BrunieV, deAndradeV, etal. Knowledge, representations, attitudes, and behaviors of women faced with taking medications while breastfeeding. J Hum Lact. 2017;98-114. https://doi.org/10.1177/0890334416679383.

11. Montgomery A, Hale TW, Academy Of Breastfeeding Medicine.. ABM clinical protocol \#15: analgesia and anesthesia for the breastfeeding mother, revised 2012. Breastfeed Med. 2012;547-53. https://doi.org/10.1089/bfm.2012.9977.

12. Bennet PN. Drugs and human lactation. Amsterdam: Elsevier; 1988.

13. Cobb B, Liu R, ValentineE, etal. Breastfeeding after anesthesia: a review for anesthesia providers regarding the transfer of medications into breast milk. Transl Perioper Pain Med. 2015;1(2):1-7.

14. Hurst N. Recognizing and treating delayed or failed lactogenesis II. J Midwifery Womens Health. 2007;588-94. https:/ / doi.org/10.1016/j.jmwh.2007.05.005.

15. Chapman DJ, Pérez-Escamilla R. Identification of risk factors for delayed onset of lactation. J Am Diet Assoc. 1999;450-4.https://doi.org/10.1016/S0002-8223(99)001091.

16. Salahudeen MS, Koshy AM, Sen S. A study of the factors affecting time to onset of lactogenesis-II after parturition. J Pharm Res. 2013;68-72. https://doi.org/10.1016/j.jopr. 2012.11.015.

17. Lind JN, Perrine CG, Li R. Relationship between use of labor pain medications and delayed onset of lactation. J Hum Lact. 2014;167-73. https://doi.org/10.1177/ 0890334413520189.

18. Rahm V-A, Hallgren A, Högberg H, et al. Plasma oxytocin levels in women during labor with or without epidural analgesia: a prospective study. Acta Obstet Gynecol Scand. 2002;81(11):1033-9.

19. Bever Babendure J, Reifsnider E, Mendias E, et al. Reduced breastfeeding rates among obese mothers: a review of contributing factors, clinical considerations and future directions. Int Breastfeed J. 2015;21. https://doi.org/10. 1186/s13006-015-0046-5.

20. Wojcicki JM. Maternal prepregnancy body mass index and initiation and duration of breastfeeding: a review of the literature. J Womens Health. 2011;341-7. https://doi.org/ 10.1089/jwh.2010.2248.

21. Takahashi K, Ganchimeg T, Ota E, et al. Prevalence of early initiation of breastfeeding and determinants of delayed initiation of breastfeeding: secondary analysis of the WHO Global Survey. Sci Rep. 2017;44868. https://doi.org/ $10.1038 /$ srep44868.

22. DeBortoliJ,Amir LH. Is onset of lactation delayed in women with diabetes in pregnancy? A systematic review. Diabet Med.2016;17-24. https://doi.org/10.1111/dme.12846.

23. Gutikova LV. Chemical composition of milk of puerperas suffered fromgestosis of different degree of severity. Biomed Khim. 2007;53(3):332-7. 
24. Hapon MB, Varas SM, Jahn GA, et al. Effects of hypothyroidism on mammary and liver lipid metabolism in virgin and late-pregnant rats. J Lipid Res. 2005;1320-30. https://doi. org/10.1194/jlr.M400325-JLR200.

25. Steer PJ. Maternal hemoglobin concentration and birth weight. Am J Clin Nutr. 2000;1285S-7S. https:// doi.org/ 10. 1093/ajcn/71.5.1285s.

26. Du G, Liu Z, Chen M, et al. Sheehan's syndrome in Xinjiang: clinical characteristics and laboratory evaluation of $97 \mathrm{pa}-$ tients. Hormones. 2015;660-7. https://doi.org/10.14310/ horm.2002.1624.

27. Drayton BA, Patterson JA, Nippita TA, et al. Red blood cell transfusion after postpartum haemorrhage and breastmilk feeding at discharge: A population-based study. Aust N Z J Obstet Gynaecol. 2016;591-8. https://doi.org/10.1111/ajo. 12485.

28. Carson JL, Guyatt G, Heddle NM, et al. Clinical practice guidelines from the AABB: red blood cell transfusion thresholds and storage. JAMA. 2016;2025-35.https://doi.org/10. 1001/jama.2016.9185.

29. Abedi P, Jahanfar S, Namvar F, et al. Breastfeeding or nipple stimulation for reducing postpartum haemorrhage in the third stage of labour. Cochrane Database Syst Rev. 2016; https://doi.org/10.1002/14651858.CD010845.pub2.

30. Jenkins IA. Tolerance and addiction; the patient, the parent or the clinician? Pediatr Anesth. 2011;794-9. https://doi. org/10.1111/j.1460-9592.2010.03501.x.

31. Hudak ML, Tan RC. Committee on drugs, et al. Neonatal drug withdrawal. Pediatrics. 2012;e540-e60. https://doi. org/10.1542/peds.2011-3212.

32. Chu TC, McCallum J, Yii MF. Breastfeeding after anaesthesia: a review of the pharmacological impact on children. Anaesth Intensive Care. 2013;41(1):35-40.

33. LarsenLA, ItoS, Koren G.Prediction ofmilk/plasma concentration ratio of drugs. Ann Pharmacother. 2003;1299-306. https://doi.org/10.1345/aph.1C379.

34. Feilberg VL, Rosenborg D, Broen Christensen C, et al. Excretion of morphine in human breast milk. Acta Anaesthesiol Scand. 1989;33(5):426-8.

35. Robieux I, Koren G, Vandenbergh H, et al. Morphine excretion in breast milk and resultant exposure of a nursing infant. JToxicolClin Toxicol. 1990;28(3):365-70.

36. Sachs HC, Committee On Drugs. The transfer of drugs and therapeutics into human breast milk: an update on selected topics. Pediatrics. 2013; e796-809. https://doi.org/10.1542/ peds.2013-1985.

37. Sauberan JB, Bradley JS, Blumer J, et al. Transmission of meropenem in breast milk. Pediatr Infect Dis J. 2012;832-4. https://doi.org/10.1097/INF.0b013e318256f4bf

38. Dean L. Codeine therapy and CYP2D6genotype.In:Medical Genetics Summaries [Internet]. Bethesda: National Center for Biotechnology Information; 2012.

39. Bodley V, Powers D. Long-term treatment of a breastfeeding mother with fluconazole-resolved nipple pain caused by yeast: a case study. J Hum Lact. 1997;307-11. https://doi. org/10.1177/089033449701300416.

40. Meyer D, Tobias JD. Adverse effects following the inadvertent administration of opioids to infants and children. Clin Pediatr (Phila). 2005;499-503. https://doi.org/ $10.1177 / 000992280504400605$.

41. Hale TW. Anesthetic medications in breastfeeding mothers. J Hum Lact. 1999;185-94. https://doi.org/10.1177/ 089033449901500302.

42. Wittels B, ScottDT, Sinatra RS. Exogenous opioids in human breast milk and acute neonatal neurobehavior: a preliminary study. Anesthesiology. 1990;73(5):864-9.
43. Al-Tamimi Y, Ilett KF, Paech MJ, et al. Estimation of infant dose and exposure to pethidine and norpethidine via breast milk following patient-controlled epidural pethidine for analgesia post caesarean delivery. Int J Obstet Anesth. 2011;128-34.https://doi.org/10.1016/j.ijoa.2010.12.004.

44. Ozkaya H, Akcan AB, Aydemir G, et al. Convulsion due to application of low dose meperidine: a case report. Agri. 2012;187-90. https://doi.org/10.5505/agri.2012.68542.

45. Schlick KH, Hemmen TM, Lyden PD. Seizures and Meperidine: overstated and underutilized. Ther Hypothermia Temp Manag. 2015;223-7. https://doi.org/10.1089/ ther.2015.0013.

46. McAuley DM, O’Neill MP, Moore J, et al. Lorazepam premedication for labour. Int J Obstet Gynecol. 1982;149-54. https://doi.org/10.1111/j.1471-0528.1982.tb04683.x

47. Ong BY, Pickering BG, Palahniuk RJ, et al. Lorazepam and diazepam as adjuncts to epidural anaesthesia for caesarean section. Can Anaesth Soc J. 1982;31-4. https://doi.org/10. 1007/BF03007945.

48. Cormack RS, Milledge JS, Hanning CD. Respiratory effects and amnesia after premedication with morphine or lorazepam. BrJAnaesth. 1977;49(4):351-61.

49. Kelly LE, Poon S, Madadi P, et al. Neonatal benzodiazepines exposure during breastfeeding. J Pediatr. 2012;448-51. https://doi.org/10.1016/j.jpeds.2012.03.003.

50. Cole AP, Hailey DM. Diazepam and active metabolite in breast milk and their transfer to the neonate. Arch Dis Child. 1975;50(9):741-2.

51. Chaves RG, Lamounier JA, César CC. Association between duration of breastfeeding and drug therapy. Asian Pac J Trop Dis. 2011;216-21. https://doi.org/10.1016/S22221808(11)60032-7.

52. Bates SM, Greer IA, MiddeldorpS, etal.VTE, thrombophilia, antithrombotic therapy, and pregnancy: antithrombotic therapy and prevention of thrombosis, 9th ed: American College of Chest Physicians Evidence-Based Clinical Practice Guidelines. Chest. 2012;e691S-e736S. https://doi.org/ 10.1378/chest.11-2300.

53. Stergiakouli E, Thapar A, Davey Smith G. Association of acetaminophen use during pregnancy with behavioral problems in childhood: evidence against confounding. Jama Pediatr. 2016;964-70. https://doi.org/10.1001/ jamapediatrics.2016.1775.

54. Damkier P, Scialli AR, Lusskin SI. Acetaminophen in pregnancy and adverse childhood neurodevelopment. Jama Pediatr.2017;396. https://doi.org/10.1001/jamapediatrics. 2016.5049.

55. Gardiner SJ, Kristensen JH, Begg EJ, et al. Transfer of olanzapine into breast milk, calculation of infant drug dose, and effect on breast-fed infants. Am J Psychiatry. 2003;1428-31. https://doi.org/10.1176/appi.ajp.160.8.1428.

56. Bonhomme V, Brichant J, Wuilmart M. Droperidol reduces nausea after caesarean section but alters the neurological status of the breastfedinfants. Abstract. The Anesthesiology annual meeting. 2002.

57. DattaS, CamannW, BaderA, etal.Clinical effects and maternal and fetal plasma concentrations of epidural ropivacaine versus bupivacaine for cesarean section. Anesthesiology. 1995;82(6):1346-52.

58. Lemas DJ, Yee S, Cacho N, et al. Exploring the contribution of maternal antibiotics and breastfeeding to development of the infant microbiome and pediatric obesity. Semin Fetal Neonatal Med. 2016;406-9. https:// doi.org/10.1016/j.siny. 2016.04.013.

59. Kirsten R, Nelson K, Kirsten D, et al. Clinical pharmacokinetics of vasodilators. Part II. Clin Pharmacokinet. 1998;9-36. https://doi.org/10.2165/00003088-199835010-00002. 
60. Kirsten R, Nelson K, Kirsten D, et al. Clinical pharmacokinetics of vasodilators. PartI.Clin Pharmacokinet. 1998;457-82. https://doi.org/10.2165/00003088-199834060-00003.

61. TheHospitalforSickChildren Toronto Ontario Canada.Motherisk [Internet]. Einsehbar unter: http:/ /www.motherisk. org. Zugegriffen:27.08.2017
62. Heck M, Fresenius M, Busch C. Repetitorium Anästhesiologie: für die Facharztprüfung und das Europäische Diplom. Berlin:Springer;2014.S.647.

63. Peer reviewed open drug database [Internet]. Einsehbar unter:http://ch.oddb.org. Zugegriffen: 16.08.2017. 